\title{
Mujeres académicas y violencia de género en una universidad de frontera
}

\section{Female professors and gender violence in a border university}

\section{ISSN 2071-8748 \\ E-ISSN 2218-3345 \\ (c) (1) $(0)$ \\ BY NC SA}

Sara Elizabeth Orellana saraelizabethorellana@gmail.com https://orcid.org/0000-0001-8470-431X

Gabriela Soria

mariagabrielasoria@gmail.com https://orcid.org/0000-0002-4147-622X

Eugenia Burgos

maruburgos13@gmail.com https://orcid.org/0000-0001-6125-7799

Ángeles Bensi angelesbensi@yahoo.com.ar https://orcid.org/0000-0002-9728-9052 CISEN-Facultad de Humanidades-UNSa

Recibido: 15/08/18 Aprobado: 12/12/18

URI: http://hdl.handle.net/11298/905

DOI: http://dx.doi.org/10.5377/entorno.v0i66.6741

\section{Resumen}

La violencia de género históricamente ha atravesado los diferentes contextos de inserción profesional de las mujeres, como un reflejo de la estructura patriarcal predominante. La universidad, como espacio social, no es ajena a esta situación. Por ello, se consideró imprescindible analizar el tema de la violencia de género contra las mujeres desde una perspectiva teórico-metodológica que considere su complejidad en los ámbitos político, cultural, social, institucional, así como de los engranajes que la constituyen en su especificidad.

Romper el ocultamiento y los silencios en torno al problema de la violencia patriarcal en todas sus formas, ponerlo en palabras desde el espacio social de la universidad, es un paso importante para visibilizarlo, analizarlo, desnaturalizarlo y prevenirlo. Desde el contexto del norte

\section{Abstract}

Historically, gender violence has permeated all spaces of professional female inclusion as a reflection of the prevailing patriarchal social structure. The university, as space for reflection, is not oblivious to this situation.

Therefore it was considered a top priority to analyze the gender violence against women from theoreticalmethodological perspective considering its complexity in the political, cultural, social, and institutional endeavors, as well as the driving gears that constitute its specificity. To break the moral cover and silence around the patriarchal violence in all its variations, to voice it out from the social spaces of the university means an important step to make it visible, to analyze it, to dissect it, and to prevent it. From the context of northern Argentina, in Salta province, this article shares the experiences of academic 
argentino, en la provincia de Salta, el presente artículo comparte experiencias de mujeres académicas respecto a tipos, modalidades, abordajes y desafíos vinculados con la violencia de género en el marco de una universidad pública de frontera.

No cabe duda de que, desde nuestra condición humana, resultan impostergables en el aquí y ahora acciones de protección para las mujeres que sufren situaciones de violencia de género, sin embargo, nuestro accionar no puede limitarse o contentarse solo con ello. Es necesario abocarnos al trabajo conjunto de soñar, pensar y construir espacios sociales más igualitarios que resquebrajen los fundamentos de la violencia de género.

\section{Palabras clave}

Educación de la mujer; Educación superior; Empoderamiento femenino; Violencia contra la mujer. women in regards with types, modalities, approaches, and challenges related with gender violence within the spaces of a public university in the Northern frontier.

There's not a slightest doubt that from our human condition it demands non-deferrable actions, here and now, to protect the women who face gender violence situations, however, our actions cannot be limited or be pleased only about it. It is necessary to contribute in the collective effort to dreaming, to thinking, and to build equalitarian social spaces that could splinter the cornerstone of gender violence.

\section{Keywords}

Women education, Higher education, Feminine empowerment, Violence against women.

\section{Introducción}

El presente escrito se enmarca en una investigación realizada desde el Centro de Investigaciones Sociales y Educativas del Norte Argentino (CISEN), perteneciente a la Facultad de Humanidades de la Universidad Nacional de Salta, en el marco de la integración de la Red de investigación en diferenciales de género en la educación superior, coordinada por la Facultad de Educación de la Universidad de Alicante, España. El objetivo del estudio fue explorar las vivencias, abordajes y desafíos que supone el tratamiento de la violencia de género en el ámbito de la Universidad Nacional de Salta, desde la mirada de las mujeres académicas que la conforman.

La Universidad Nacional de Salta (UNSa), es una institución de gestión pública, ubicada en la provincia de Salta, en el norte de Argentina. Se trata de una universidad joven, su origen se sitúa en 1972. Sus actividades académicas se organizan en una Sede central, ubicada en la ciudad de Salta y en tres Sedes regionales, desplegadas en el interior de la provincia: 1) Sede Regional Orán; 2) Sede Regional Tartagal y 3) Sede Regional Sur (Metán y Rosario de la Frontera). Desde su fundación, la UNSa fue concebida como "Regional y
Latinoamericana", con la exigencia de desempeñar un papel protagónico en las reformas socioeconómicas y culturales que eran imprescindibles en la región, asumiendo como lema original: "Mi sabiduría viene de esta tierra".

Sostenemos que, en el mapa de las universidades públicas argentinas, nuestra institución, es una universidad de frontera, no sólo en términos geográficos sino fundamentalmente simbólicos, en tanto "locus" de enunciación diferencial, que significa un desplazamiento de los conceptos y de las prácticas colonizantes. Universidad de frontera que más que límite, se presenta como horizonte, que posibilita un diálogo plural y polifónico entre los/as que la habitan, tensionando las racionalidades cotidianas. La pensamos como una universidad que crece en las fronteras, en los resquicios, en los extramuros y desde allí, el desafío por desnaturalizar entre otras, la violencia de género que históricamente ha atravesado los diferentes contextos de inserción profesional de las mujeres y de la cual la universidad como espacio social, no queda exenta.

En torno a ese "locus" de enunciación diferencial, afirmamos que la violencia de género históricamente ha atravesado los diferentes contextos de inserción profesional de las 
mujeres, reflejo de la estructura patriarcal predominante. Comprendemos que hablar de violencia de género se fundamenta en "las distintas formas de violencia contra las mujeres, pero también en otras violencias que tienen origen en las relaciones de poder y desigualdad que se fundan en el sistema sexo-género-sexualidad" a decir de González Prado y Yanes Betancour (2013: 53).

La violencia suele hacerse presente en relaciones de desigualdad, de asimetría, en donde, por un lado, se encuentra un sujeto en una posición y condición de superioridad y, por el otro, alguien en posición y condición de subordinación. En el presente trabajo optamos por un posicionamiento que aborda la violencia como problema de los derechos humanos, que nos permita visualizar las posibles agresiones, violencias o embates que las mujeres académicas suelen vivir y padecer en el ámbito laboral universitario. El que las mujeres vivamos diferentes situaciones de violencias a lo largo de nuestras vidas se traduce en un hilo conductor común en la experiencia vital (subjetiva y social) que compartimos con otras congéneres. Ello da cuenta del carácter transversal, interclasista, universal, que asume esta realidad que amenaza los derechos de la mitad de la población de la humanidad.

La violencia contra las mujeres constituye un problema de derechos humanos, un fenómeno multidimensional cuyas aristas hunden sus raíces hasta los niveles más profundos y subterráneos de las tramas sociales, culturales, políticas e históricas de la agitada contemporaneidad que transitamos como sociedad. Estamos ante una realidad que si bien reconocemos como de larguísima data, en la actualidad reviste nuevas facetas, dejando al descubierto las resignificaciones que la misma asume en las relaciones de género que desplegamos mujeres, hombres y otras identidades sexo-genéricas.

\section{Metodología}

En el marco de la Red de Investigación en Diferenciales de Género en la Educación Superior, coordinada por la Facultad de Educación de la Universidad de Alicante, España, venimos desarrollando desde el año 2014, trabajos de investigación exploratorios y cualitativos, en torno a diferentes aspectos de Ios diferenciales de género en el contexto de la Universidad Nacional de Salta y, sobre todo, en el ámbito de la Facultad de Humanidades en la sede central (Salta-Capital) y en la sede (Tartagal- Norte de la provincia de Salta), unidades académicas de pertenencia de este equipo.

Resultado de este proceso investigativo podemos mencionar los siguientes trabajos realizados y socializados en los Encuentros anuales de la Red: "Mujeres en la gestión universitaria desde una universidad de frontera" (Quito, 2017); "Universidad y diferencia: polifonías juveniles" (Cochabamba, 2016); Diferencias y desigualdades de género: voces de mujeres académicas en una universidad de frontera" (Bogotá-Colombia, 2015); "Universidad Nacional de Salta: un diagnóstico inicial para actuaciones por la igualdad en las diferencias" (Maule-Chile, 2014).

Desde esos antecedentes, el presente estudio exploratorio y cualitativo tuvo como objetivo general indagar sobre la violencia de género que afecta a las mujeres académicas en el contexto de la Universidad Nacional de Salta. En tanto objetivos específicos establecimos: Describir situaciones de violencia de género que vivencian las académicas y analizar las perspectivas de las participantes respecto a ella; Conocer las normativas vigentes en materia de violencia de género e indagar sobre modalidades de actuación desde la perspectiva de las participantes.

Este trabajo se inscribe en la perspectiva crítica de la investigación socioeducativa, es decir, la metodología de investigación que rebasa la dimensión técnica 0 instrumental $y$, se entiende como un conjunto articulado de herramientas e instrumentos que permiten pensar problemas relevantes y construir los procedimientos que conduzcan a su comprensión / explicación / interpretación en su contexto histórico, social, cultural y político. En este sentido, cada realidad y problema de investigación demanda una interacción constante y dialéctica entre la teoría, el método, el trabajo de campo, los datos obtenidos y el problema (Rockwell, 2009).

Para ello, realizamos encuestas y entrevistas a mujeres académicas que ocupan diferentes posiciones en nuestra universidad, a fin de conocer aspectos referentes a las situaciones de violencia de género en el cotidiano hacer de este contexto de universidad de frontera. El universo de estudio se constituyó de mujeres académicas de distintas unidades académicas de la UNSa, conformando el siguiente perfil: 
- $\quad$ El $80 \%$ pertenece a la Sede Central de la Universidad y el $20 \%$ a la Sede Tartagal.

- Según cargos que desempeñan, en su mayoría se ubican en el estamento de auxiliares de la docencia universitaria sea como Jefas de Trabajos Prácticos o bien como Auxiliares de Docencia de Primera Categoría. Solo un $30 \%$ posee cargo de Profesora responsable de cátedra.

- El $30 \%$ corresponde a docentes noveles (hasta 10 años de antigüedad) y el $70 \%$ a docentes expertas (entre 13 y 30 años de antigüedad).

- El 30 \% se desempeña en Ciencias Exactas e Ingeniería; El $30 \%$ se desempeña en Ciencias Sociales y Humanidades; El 30 \% se desempeña en Ciencias de la Salud; un $10 \%$ no responde a este dato.

- El $33 \%$ posee título de posgrado.

- Rango etario: 50 \% tienen entre 40 y 45 años y el $50 \%$ restante, entre 51 y 59 años.

A continuación, socializamos algunos de los resultados del estudio referidos a aspectos relevantes que posibilitan presentar el estado de situación de nuestra universidad. Para ello, presentamos tipos y modalidades de violencia de género, marcos reglamentarios, modalidades de actuación y desafíos en torno a la misma. También y desde una opción de posicionamiento de quienes escriben, recuperamos expresiones textuales de las entrevistadas sosteniendo que ellas pueden colaborar a la desnaturalización, al desocultamiento de prácticas institucionales que suelen pasar inadvertidas, justificadas en algunos casos, desde los patrones del patriarcado.

\section{Resultados y Discusión}

La Universidad Nacional de Salta (UNSa), se inscribe en el contexto de la provincia de Salta, en el norte de Argentina. No resulta menor que en el año 2014, el gobierno provincial declaró la emergencia social por violencia de género en todo su territorio. Salta, se encuentra en segundo lugar a nivel nacional por femicidios. Los datos, con el consecuente subregistro que involucran, son alarmantes y exponen la beligerancia de esta problemática'.

La visibilización y la denuncia respecto de las múltiples formas de violencia que se multiplican y afectan a niñas, jóvenes y mujeres adultas requiere ser reconocida como un problema social que afecta a todos/as, dejando de ser pensado como un asunto que se limita a la esfera de la vida privada. Aunque es sabido que subsiste socialmente la idea de impenetrabilidad del espacio privado de las personas sobre la que es necesario seguir colisionando. Ya en los años 90 los trabajos de la feminista Christine Delphy problematizaba la constitución de la esfera privada como una esfera de no derecho para las mujeres. (Falquet, 2017).

\section{Tipos y modalidades de violencia de género en el} ámbito universitario

La UNSa, única universidad pública de la provincia de Salta, no es ajena al contexto que la alberga. Consultadas sobre situaciones de violencia de género en el ámbito universitario la mayoría de las mujeres académicas, expresan categóricamente conocer o haber sido víctimas de casos de violencia en esta institución. Cabe mencionar que también ellas, refieren a una doble esfera, la pública (aquellas situaciones que toman conocimiento públicoinstitucional) y, la esfera privada (aquellas situaciones que no trascienden en su conocimiento $o$ al menos se reduce a comentarios informales, entre poco/as).

Si tomamos como referente la Ley Nacional $N^{\circ} 26,485$, de Protección Integral para prevenir, sancionar y erradicar la violencia contra las mujeres en todos los ámbitos en que desarrollan sus relaciones personales, sancionada en Argentina en el año 2009, podemos observar como en su artículo $\mathrm{N}^{0} 5$ y 6 se puntualizan distintos tipos y modalidades de violencia contra las mujeres. Clasifica en tipos de violencia: 1) La física; 2) La psicológica; 3) La sexual; 4) La económica y patrimonial; y 5) La simbólica. Respecto a las modalidades o sea las formas en que se manifiestan los distintos tipos de violencia contra las mujeres en los diferentes ámbitos, se señala lo siguiente: 1) Violencia doméstica; 2) Violencia institucional; 3) Violencia laboral; 4) Violencia contra la libertad reproductiva; 5) Violencia obstétrica; 6) Violencia mediática.

En este sentido, como resultado del estudio podemos expresar que los casos denunciados por las docentes de la muestra se corresponden a modalidades de violencia institucional y laboral contra las mujeres, donde identificamos claramente la prevalencia de tipos de violencia simbólica y psicológica, aunque esto no descarte, otras como la física o sexual, expresadas en menor frecuencia.

\footnotetext{
1 Para mayor profundización de datos ver Informes Anuales 2016-2017 del OVcM de Salta.
} 
En el análisis de los datos, observamos que, en las situaciones de violencia de género mencionadas por las mujeres de nuestro contexto universitario, confluyen diferentes tipos de violencia en forma simultánea. Podemos reafirmar entonces, que en las modalidades de violencia institucional y laboral, la violencia simbólica y psicológica prevale y actúan en forma conjunta, en la mayoría de los casos presentados. Las siguientes proposiciones intentan ilustrar, ya que tienen recurrencia y frecuencia en lo relatado por nuestras académicas.

Explotación laboral al interior de equipos de cátedras conformados por mujeres y hombres. Sobrecarga de tareas para la mujer académica, justificada en estereotipos de género;

Expresiones verbales de desvalorización de la mujer en espacios de intercambio intracátedra, de gestión institucional y en situaciones de elecciones democráticas, sostenido también en estereotipos de género;

Patrones culturales como justificativos ante hechos de violencia verbal o de relaciones desigualitarias. "Lo dice en broma"; "Los hombres son así"; "En Salta es común"; Discriminación de parte de docentes en cursado de materias, privilegios para estudiantesvarones, justificado en estereotipos de género según desempeños en áreas de conocimiento.

En este proceso de visibilización de la violencia de género recuperamos también, expresiones textuales de los testimonios de las docentes entrevistadas. Consideramos que ellas pueden contribuir a la desnaturalización, al desocultamiento de prácticas en el ámbito universitario, que asentadas en una cultura institucional no ajena a lo social en la que se inscriben, muchas veces son inadvertidas y hasta justificadas desde los patrones del patriarcado.

"Tengo presente la situación de violencia generada hace unos cuantos años en el marco de un conflicto entre la facultad de Humanidades y el rectorado de la Universidad. Nuestra decana era mujer y el rector, varón. Recuerdo las formas irrespetuosas, inadecuadas y descalificativas que el rector utilizaba para referirse a la decana, intentando de esa manera resquebrajar el posicionamiento político que nuestra decana representaba". (Prof. en Ciencias de la Educación, 17 años de antigüedad)
"Maltrato de su jefe, en esos momentos Decano de la facultad. Firmamos una nota en apoyo a esta compañera y la presentamos en la facultad. El propio Consejo Directivo hizo un mamarracho en el tratamiento de la citada nota, cuestión que la compañera terminó retirando la denuncia $y$ 'negociando' con el victimario. Un desastre, creo que para salvarse el pellejo el victimario y desalentar cualquier denuncia por el estilo". (Ingeniera Industrial, 30 años de antigüedad)

"El caso más claro es el de algunos hombres que hacen como si las voces femeninas no existieran y le quitan performatividad. Esto sucede entre estudiantes, entre docentes y auxiliares y con el personal de apoyo. Si hay una posición de jerarquía del varón, puede agravarse, pero muchas veces, se da entre pares o la jerarquía institucional no tiene la importancia que tiene la de género". (Doctora en Letras, 30 años de antigüedad)

"Recientemente, en situación electoral un docente agredió verbal e intimidó físicamente a una candidata de la lista opositora. Los pares niegan la desmesura de la situación, pero las afectados hicieron las denuncias institucionales $y$ ante la Justicia que corresponden". (Doctora en Letras, 30 años de antigüedad)

"Sí, lo viví en la carrera de analista en sistemas, de parte de dos docentes masculinos que tenían varias asignaturas de informática a cargo: discriminaban a las mujeres, nos ignoraban y nos maltrataban expresándose en forma despectiva y burlona por nuestra condición de ser mujer no apta para ese tipo de carrera y sobre todo jugaban con nuestras notas en instancias de exámenes, estas situaciones fueron muy torturantes". (Lic. en Análisis de sistemas, 25 años de antigüedad)

"Conozco varios casos de violencia de género, tales como bromas y descalificaciones a docentes mujeres en el trabajo cotidiano. Un docente que agredió a la candidata mujer de la lista opositora y no así a los varones de la misma lista por ejercer su derecho a ser fiscal en una elección de directores y sobre todo por ser mujer". (Lic. en Nutrición, 10 años de antigüedad)

"En la cátedra en la que me desempeño he vivido mucho maltrato psicológico, inclusive el colmo fue cuando el 'jefe' 
me gritó frente a los estudiantes que rendían un parcial. Esto me ocasionó siete derrames sucesivos en los ojos, no había un reconocimiento al tremendo esfuerzo de sacar adelante a entre 60 y 100 estudiantes en el cuatrimestre". (Ingeniera Industrial, 30 años de antigüedad)

"Hace algunos años, interpelé a un docente que estaba amenazando a una consejera estudiantil del consejo Directivo con el cuerpo y con palabras para que votara su moción. Por la misma época fui amenazada por estudiantes varones de (...) para que aceptara que rindieran un parcial estudiantes que ya estaban libres. Me rodearon cerca de 15 muchachos y me apretaban verbal y literalmente tratando de impedir que tomara el examen. Se tomó igual y tampoco cedi ante sus amenazas. Luego traté de hablar con ellos para hacerles analizar su actitud sin mucho éxito". (Doctora en Letras, 30 años de antigüedad)

"Otra compañera, (...) hizo una denuncia en la facultad referida al maltrato de su jefe, en esos momentos decano de la facultad. Firmamos una nota en apoyo a esta compañera y la presentamos en la facultad. El propio Consejo Directivo hizo un mamarracho en el tratamiento de la citada nota, cuestión que la compañera terminó retirando la denuncia y 'negociando' con el victimario...". (Magister en Recursos Naturales y Medio Ambiente, 30 años de antigüedad)

“(...) Tanto en mi caso como el de compañeras de la facultad que se sintieron violentadas por las prácticas de los colegas. Incluso uno de los casos fue de acoso sexual, esta compañera enfermó gravemente poniendo en riesgo su salud y estabilidad emocional y laboral porque quería renunciar. Hizo la denuncia verbal en decanato y no se resolvió nada". (Magister en Recursos Naturales y Medio Ambiente, 30 años de antigüedad)

Estas voces y testimonios de colegas nos alertan y movilizan, reafirmando la urgencia de acciones sistemáticas como agentes sociales comprometidas/os con la eliminación de la violencia hacia las mujeres, para lo cual reconocemos como ineludible la tarea de cuestionamiento y revisión de patrones de pensamiento, así como, los modos de abordaje de la temática desde los distintos campos disciplinares al interior de la praxis concientizadora y crítica que la universidad pública promueve.
Consideramos que es preciso pensar y visibilizar la violencia contra las mujeres como interclasista, intergeneracional, oblicua porque es resultado de un determinado modelo de poder. Además, reviste un evidente problema político, al configurarse en sí misma como un problema de poder, de desigualdad estructural y de jerarquización de un colectivo (varones) sobre otro (mujeres). Por ello creemos imprescindible analizar el tema de los derechos humanos y el de la violencia de género contra las mujeres desde una perspectiva que considere lo político, cultural, lo social, lo institucional, entre otros aspectos, provocando reales cambios en la distribución desigual del poder en las sociedades.

La violencia contra las mujeres excede completamente la caracterización como un hecho de dificultad en las relaciones entre sujetos. Da cuenta de una determinada división sexual del trabajo, de relaciones de poder asimétricas entre mujeres y varones, y de la permanencia de privilegios para estos, situaciones todas que impide el ejercicio de la ciudadanía de las mujeres en igualdad de condiciones².

\section{Sobre el abordaje de la violencia de género en el marco institucional}

Las docentes también se manifestaron en relación con cómo abordar la violencia de género en el campo universitario, diferenciando acciones en distintas dimensiones de la vida universitaria e identificando órganos o herramientas para estas.

\section{Desde lo académico-administrativo:}

Integrar a la formación profesional que ofrece la universidad, el tema de la violencia de género, a derechos humanos. "Los países latinoamericanos en particular, necesitan contextualizar esa formación en el paradigma de descolonización a raíz de instalación del patriarcado"

Sensibilizary capacitar en el tema tanto aadministrativos, docentes, estudiantes y autoridades.

Implementar seminarios, cátedras abiertas.

Que la institución universitaria fije posición sobre el tema de la violencia de género.

Revisar normativas que revisten lenguaje sexista.

\footnotetext{
2 González Patricia; Yanes Betancour, A. (coord.) 2013 "Violencia contra las mujeres. Quien calla otorga". IDEGE UNCuyo - INCIHUSA CONICET- DESCOSIDAS. Mendoza, Argentina.
} 
Desde la asistencia gremial, jurídica y normativa:

Las académicas expresan el deseo/necesidad de visibilizar la violencia de género en el ámbito universitario, para que puedan existir posibilidades reales de acción y transformación.

Por ello priorizan:

Contener y animar para que las mujeres violentadas realicen la denuncia ante las autoridades de la universidad, Comisión de la mujer de la universidad y gremio docente.

Denunciar a línea gratuita 144 (de contención y asesoramiento ante situaciones de violencia).

Solicitar asesoramiento jurídico y patrocinio legal.

\section{Conocimiento de Marcos normativos} relativos a la violencia de género

El marco legal argentino persigue promover y garantizar la eliminación de toda forma de discriminación entre mujeres y varones a través de la remoción de patrones socioculturales y barreras económicas que promueven y sostienen la desigualdad de género. Indagadas respecto a marcos reglamentarios nacionales, provincial e institucional, las mujeres académicas en un $50 \%$ expresaron desconocer las normativas vigentes. El grupo restante precisó que conocía algunas leyes y entre las más referenciadas señalaron:

Ley Provincial № 7888 de Protección a Víctimas de Violencia de Género (2015).

Ley Provincial № 7403 de Violencia Familiar (2004).

Ley Nacional № 26.485 de Protección Integral para prevenir, sancionar y erradicar la violencia contra las mujeres (2009).

Ley Nacional № 26.618 de Matrimonio Igualitario (2010). Ley Nacional № 26.743 de Identidad de Género (2012).

Ley Nacional $N^{\circ} 25.087$ de Delitos contra la Integridad Sexual (1999).

Ley Nacional № 26.150 de Educación Sexual Integral (2006).

Ley Nacional № 26.842 contra la Trata de Personas (2012).

La totalidad de las mujeres de la muestra coinciden en afirmar que consideran relevante que estas normativas sobre la Violencia de Género y la discriminación se difundan e implementen a nivel institucional en la universidad.
Respecto a ello, expresa una de las docentes entrevistada: "Considero que se deben difundir en la institución y ser objeto de promoción a través de específicas campañas para lograr crear situaciones de debate y de conocimiento crítico de las mismas, tendientes a reflexionar sobre las conductas de todos los miembros de la comunidad universitaria que favorecen o distancian de la conformación de un ámbito institucional respetuoso..."

\section{Protocolo en Violencia de Género en el ámbito universitario}

El $92 \%$ de las entrevistadas respondió afirmativamente a la consulta sobre si consideran necesaria una normativa específica para el ámbito universitario. Las respuestas negativas mencionaron que no creen necesaria una norma específica a la Violencia de Género, sino más amplia a todo tipo de violencias.

Entre las expresiones más significativas recuperamos:

"Es necesario un Protocolo para la intervención en estas situaciones".

"Sí. Es muy necesario con ajuste a las Leyes nacionales que han logrado sancionarse en nuestro país. Esta acción afianza la demanda y aplicabilidad de las leyes para que transformen la mentalidad patriarcal y colonizadora en las que se sostienen las prácticas de violencia de género".

"Absolutamente sí, me parece urgente trabajar en este sentido".

"Sí creo muy necesario, porque ello implicaría visibilizar la problemática que atraviesan las instituciones educativas en el nivel superior y de esta manera promover prácticas académicas realmente democráticas y plurales".

"Sí, es necesario, ya que no tenemos un respaldo universitario. La normativa nos protegería y nos daría un aliento para accionar, tenemos que saber las medidas preventivas y decisivas compatibles con el proceso legal. Serán herramientas útiles para enfrentar situaciones de violencia en este ámbito,...que estas normativas sean claras y específicas".

"Existen ya en otras universidades y me parecen muy positivas... son herramientas para un cambio en la universidad".

"Importante para evitar todo tipo de violencia institucional, no solo la de género". 
"Me encantaría participar de una Comisión que diseñe, implemente y cuide la aplicación de normativas en este sentido".

"Lo necesitamos porque afecta a un número importante de docentes".

Lo expresado hasta aquí da cuenta de cómo la violencia de género penetra en sus distintas manifestaciones y modalidades el ámbito universitario y que, sin duda, afecta a las otras mujeres que lo conforman en su entramado de relaciones: mujeres estudiantes, mujeres personales de apoyo universitario, entre otras.

También es claro que, hacer visible la existencia de la violencia de género en nuestra universidad, es solo el primer paso de un camino por recorrer. Continúa un proceso difícil, pues se trata de desnaturalizarla, de resquebrajarla en sus fundamentos existenciales que es el nivel de las estructuras, al decir de (Bourdieu, 1995) "estructuras estructurantes estructuradas", el habitus, individual pero social al mismo tiempo y que no pocas veces, es la principal causa de la reproducción de los principios de percepción y acción que desigualan las relaciones entre mujeres y varones y que conllevan a la violencia de género.

\section{Conclusiones}

La sociedad en la que vivimos ha naturalizado históricamente- la violencia hacia las mujeres y sus múltiples expresiones a lo largo de los tiempos. Si bien los discursos de la opinión pública, los medios de comunicación, las representaciones sociales acerca del tema están cambiando paulatinamente y se registra un grado de sensibilización y atención mayor ante esta problemática, en las prácticas, se mantiene férreo un modo de control social hacia las mujeres $y$, en ese sentido, nociones como las de "normalidad", "naturalidad" se hacen presentes al debatir la temática.
Romper el ocultamiento y los silencios en torno al problema de la violencia patriarcal en todas sus formas, ponerlo en palabras en los diferentes espacios institucionales, es un paso importante para visibilizarlo, analizarlo, desnaturalizarlo y prevenirlo. Puntualmente, en la cotidianidad de la universidad, tienen lugar procesos de construcción de un orden pedagógico que contribuye a definir y conformar sujetos femeninos y masculinos a través de la transmisión de un caudal específico de definiciones, relaciones, roles y normas de género, que pautan maneras de comportamiento aceptadas -y no- para cada género.

Sostenemos entonces, que la universidad tiene el enorme potencial de aportar a la transformación de las bases culturales, modificando concepciones y prácticas que se reproducen a través de las matrices simbólicas y promoviendo iniciativas orientadas a generar mayores condiciones para la igualdad de derechos. En consonancia con ello, la universidad, como otras instituciones formadoras, es entendida como un ámbito protector y promotor de derechos, tiene la responsabilidad de intervenir frente a todo tipo de vulneración de estos en sus agentes (maltrato, violencias, discriminación, abusos y acoso sexual, violencia institucional, entre otros).

La transformación social que exige el respeto de los derechos humanos de las mujeres precisa situarnos -y situarlasen el centro de los cambios que resultan ineludibles, comprendiendo las diferentes formas de pensar, sentir, actuar y existir de las mujeres en la sociedad. Una mirada compleja deja abierta la inquietud sobre aspectos menos visibles de las relaciones de género en el contexto universitario local: cómo ellas construyen y reconstruyen sentidos de vida, emblemas identificatorios, vínculos interpersonales y sociales desde una universidad de frontera. Ante ello, asumimos, un desafío prioritario, aportar a la institucionalización de la perspectiva de género, esto es, afianzar el proceso político mediante el cual se legitima la necesidad de establecer políticas y acciones dirigidas a superar desigualdades entre los géneros y en el interior de ellos. 


\section{Referencias}

Bourdieu, P. \& Passeron, J. C. (1995). La reproducción. México: Fontamara.

Camacho, R. (2003) ¿Qué son los derechos humanos de las mujeres? En Acercándonos a los instrumentos internacionales de protección de los derechos de las mujeres. San José de Costa Rica: Instituto Interamericano de DD.HH. -Asdi - DANIDA.

Chiarotti, S. (2010). Derechos humanos de las mujeres. En S. Chiarotti. Derechos Humanos de las mujeres. Recursos y mecanismos de reclamo y monitoreo. Santa Fe, Argentina: Instituto de género, derecho y desarrollo (INSGENAR).

Espinar Ruiz, E. (2007). Las raíces socioculturales de la violencia de género. Dpto. de Sociología II Universidad de Alicante. Escuela Abierta, ISSN 1138-6908, 10, 23-48

Falquet, J. (2017). Pax neoliberalia. Perspectivas feministas sobre (la reorganización de) la violencia contra las mujeres. Buenos Aires: Madreselva.

Femenías, M. L. (2008). Articulaciones sobre la violencia contra las mujeres. Urdimbres que marcan la trama. En E. Aponte Sánchez \& M. L. Femenías (comps.) Articulaciones sobre la violencia contra las mujeres. La Plata Buenos Aires: EDULP.
González Prado, P. \& Yanes Betancour, A. (2013). Violencia contra las mujeres y violencia de Género. La cuestión terminológica y los paradigmas implicados. En P. González Prado \& A. Yanes Violencia contra las mujeres. Quien calla otorga. Mendoza, Argentina: IDEGE -Descosidas- CONICET UNCuyo.

OVCM Observatorio de Violencia contra las Mujeres. (2016). Marco conceptual y normativo. En Informe Anual. Recuperado de http//www.ovcmsalta.gob.ar.

Ley Nacional $\mathrm{N}^{\circ} 26.485$ (2009). Protección integral para prevenir, sancionar y erradicar la violencia contra las mujeres en todos los ámbitos en que desarrollan sus relaciones interpersonales.

Ley Provincial № 7888 (2015). Protección contra la violencia de género. Salta, Argentina.

Rockwell, E. (2009). La experiencia etnográfica: historia y cultura en los procesos educativos. $1^{\circ} \mathrm{Ed}$. Buenos Aires: Paidós

Rodigou Nocetti, M. (2011). Violencia hacia las mujeres: entre la visibilización y la invisibilización. En A. Domínguez \& A. Morcillo (comps.). Derechos humanos, género y violencias. Córdoba Argentina: UNC.

Velázquez, S. (2007). Violencias cotidianas, violencia de género. Escuchar, comprender, ayudar. Buenos Aires Argentina: Paidós 